\title{
Local Photorefractive Modification in Lithium Niobate Using Ultrafast Direct Laser Write Technique
}

\author{
Domas Paipulas $^{1,3}$, Ricardas Buivydas ${ }^{2}$, Saulius Juodkazis ${ }^{2}$ and Vygantas Mizeikis ${ }^{1}$ \\ ${ }^{1}$ Laser Research Center, Department of Quantum Electronics, Physics Faculty, Vilnius University, Lithuania \\ ${ }^{2} 2$ Centre for Micro-Photonics, Swinburne University of Technology, Australia \\ ${ }^{3}$ Research Institute of Electronics, Shizuoka University, Japan \\ dvmzks@gmail.com
}

\begin{abstract}
In this work we demonstrate the possibility to form discrete homogeneously-altered refractive index regions of photorefractive origin in bulk of pure and iron doped lithium niobate crystals using femtosecond direct laser write technique. We show that nonlinear free charge generation and charge separation caused by the bulk photovoltaic effect are the main contributing factors to the change in refractive index. Moreover, femtosecond pulse induced refractive index change can be by an order of magnitude higher than values reached with longer laser pulses. Additionaly, we show that with different recording algorithms a sufficient control on topological charge separation can be achieved in micrometer scale that lets implement reversible information recording procedures in lithium niobate crystal.
\end{abstract}

DOI: $10.2961 / \mathrm{jlmn} .2016 .02 .0016$

Keywords: femtosecond microfabrication, direct laser write, photorefractivity, lithium niobate

\section{Introduction}

Lithium niobate $\left(\mathrm{LiNbO}_{3}, \mathrm{LN}\right)$ is one of the most important crystalline materials used in photonics applications. LN is valued for its excellent ferroelectric, piezoelectric, pyroelectric properties and strong optical nonlinearity. This diverse material proved itself in many applications such as frequency converting, optical signal modulation (electro-optical and acousto-optical), waveguide integration and etc [1].

One property of LN that stands out from other ferroelectric materials is a very strong photorefractive effect. Nonhomogeneous illumination at low light intensities (as low as $10 \mathrm{~mW} / \mathrm{cm}^{2}$ for visible light) induces local changes in refractive index; however these variations as a rule are not permanent and can be optically or thermally nullified without any residual change in the host crystal $[2,3]$. Depending on the application material photorefractivity could be beneficial or harmful: former case illustrates the widespread use of LN as a medium for holographic recording [1], while low photorefractive damage value can sufficiently degrade laser beam when crystal is used for nonlinear applications [3]. It is generally accepted that photorefractivity is a well understood phenomenon at low light intensities [2] while recent decade showed great progress in describing its behavior and nuances at high light intensities for doped and nominally pure crystals $[4,5,6]$.

Localized modification of optical properties in wide range of transparent (not necessarily photosensitive) materials become possible with the progress of femtosecond laser systems [7]. Nonlinear absorption effects initiated with focused laser beam allow three-dimensional integration of (micro)photonic devices in transparent materials by Direct Laser Writing (DLW) technique or similar methods [8]. The greatest attention was granted to microfabrication of glasses, but successful integration of permanent and quasi-permanent waveguides $[9,10,11,12]$, volumetric diffractive optics elements [13], planar devices [14] in LN crystal were also reported. In these studies integration part was realized through precise control of microdamage formation, where refractive index change is induced through material amorphization or stress-driven effects which result in permanent structure development [15]. Photorefractive aspect in femtosecond DLW was scarcely investigated. It was showed experimentally and theoretically that femtosecond laser pulses can induce photorefractive effect through non-linear absorption without catastrophic damage of the LN crystal[16, 17]. Signs of photorefractive behavior in some laser-written waveguides were also reported [11], as well as an ability to record photorefractive data bits in 3D volume of Fe doped LN crystal [18]; however, the potential of applying photorefractivity effect in femtosecond DLW is not yet clearly identified.

In this work we demonstrate that femtosecond DLW technique can be easily applied for photorefractive modification inscription in the Fe doped and nominaly pure LN crystal. Photorefractive modifications are induced in the proximity of laser focus via nonlinear absorption effect and threedimensional optical structures can be realized without material damage. In addition, reversible nature of induced modifications can be exploited for dynamic data recording in 3D space.

\section{Induction of photorefractive modifications with fo- cused laser beam}

Photorefractivity is a change of refractive index caused by the linear electrooptic (Pockels) effect. This mechanism could be explained in the following process chain: photoexitation creates free charge carriers by shifting electrons from valence to conduction band; these carriers diffuse or drift from the excitated area (spatial charge transport); finally, 
free carriers are trapped by acceptors at different crystal location, yielding the development of uneven space-charge distribution which is accompanied with intrinsic space-charge electric field. This electric field causes changes in refractive index via Pockels effect.

Several physical mechanisms can be responsible for charge transport: drift, diffusion, and Bulk Photovoltaic Effect (BPVE). Drift is an ohmic current which appears when free-charge is put in an electric field: such field could be externally applied or be present in a crystal due to its properties, i.e. pyro/piezoelectric effects. Diffusion currents arise when a charge density gradient is present in the crystal - a common situation when photoexcitation is produced with inhomogeneous radiation. Finally, BPVE appears in non-centrosymetrical crystals due to asymmetric photoexcitation probabilities: excitation in one spacial direction is more probable than in others which causes the appearance of ballistic current where free-charges have non-zero net momentum along one direction [19]. A combination of these charge transport mechanisms forms the complete picture of space-charge separation in the medium.

In LN the dominant charge separation mechanism is BPVE. It was demonstrated, that pyroelectric drift and diffusion are capable to induce space-charge fields that are by two orders lower than BPVE alone [20,3]. This means that a specific direction ( $c$-axis) exists where main charge transport takes place.

a)

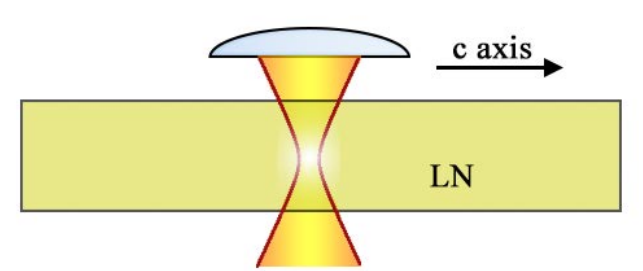

b)

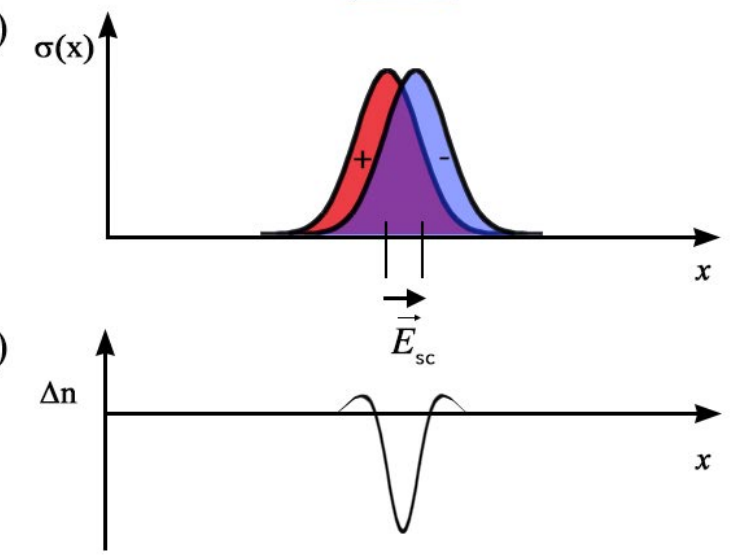

Fig. 1. a) Recording of photorefractive modification with focused laser beam; b) schematic one-dimensional description of the spacecharge field formation' c) local reduction of refractive index via Pockels effect.

Space-charge distribution creates space-charge field $\left(E_{s c}\right)$ which causes change in refractive index according to
Pockels equation:

$$
\Delta n_{o, e}=-\frac{1}{2} n_{o, e}^{3} r_{13,33} E_{s c}
$$

here $n_{o, e}$ is ordinary or extraordinary refractive indexes, and $r_{13,33}$ is electrooptical coefficients (for LN these are $11 \mathrm{pm} / \mathrm{V}$ and $34 \mathrm{pm} / \mathrm{V}$ [21]) while $E_{s c}$ is directed along $c$-axis. Electric field values induced duo to BPVE can reach up to $10 \mathrm{MV} / \mathrm{mm}$ in LN:Fe yielding a refractive index change up to $\sim 10^{-3}$.

Focusing femtosecond radiation inside the bulk of the LN crystal allows to reach intensities high enough to initiate multiphoton absorption as well as induce photomodification inside the volume of material. As charge mean travel length is much smaller than laser focus spot size, the high spatial control of the photomodified area can be achieved, as shown in Fig.1).

Induced refractive index change in the material is determined by the internal space-charge electric field. The magnitude of this field depends not only on the quantity of the separated charge, but also on the topological nuances. Consider two cases depicted in Fig. 2-a,b).

In the first case laser beam is translated perpendicularly to $c$-axis, while in the second case the beam translation is parallel. As charge separation appears only along $c$-axis, resulting charge distribution will be different. In perpendicular case positive and negative charge will be arranged symmetrically on both sides of laser written line, while in parallel case, positive and negative charges will separate along laser written line. The resulting intrinsic electric field will be completely different in these two cases. In Fig. 2-a,b) it is shown a modeled refractive index distribution with separated charge presence for both cases. Modeling was carried out by solving 2D Poisson's equation for separate cases using Finite Difference Method (FDM) which gave electric field distribution in the crystal; the refractive index change then was evaluated with Pockels formula (1). The magnitude of refractive index change was normalized.

Note that for a single drawn line, the refractive index modulation across the modification is negative, and the modulation is strongest when sample is translated perpendicularly to $c$-axis (Fig. 2-a)). Only the edges of modification posses increased refractive index at places were electric field changes orientation. When the sample is translated along the $c$-axis, refractive index modulation at the center becomes negligible and only edges experience sufficient modulation (Fig. 2-b). This effect can be employed for dynamic data recording/erasing, which is sketched in Fig. 2c).At first, perpendicular-to- $c$-axis line is inscribed, yielding a strong electric field and high magnitude of refractive index change in the material (data recording process); afterwards, if parallel-to- $c$-axis line is written on top of previous modification, charge will be redistributed and newly formed electric field screens previous modification resulting in lower refractive index change. This processes is an analogous to dataerasure procedure. While charge separation is not saturated, recording/erasure cycle could be repeated many times.

Eventually, for ultrafast DLW all these mentioned inscription strategies could be realized in 3D space. 


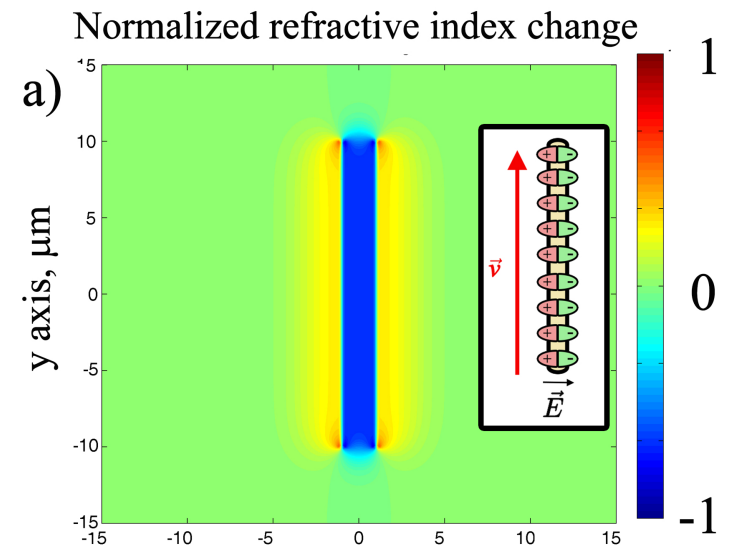

b)
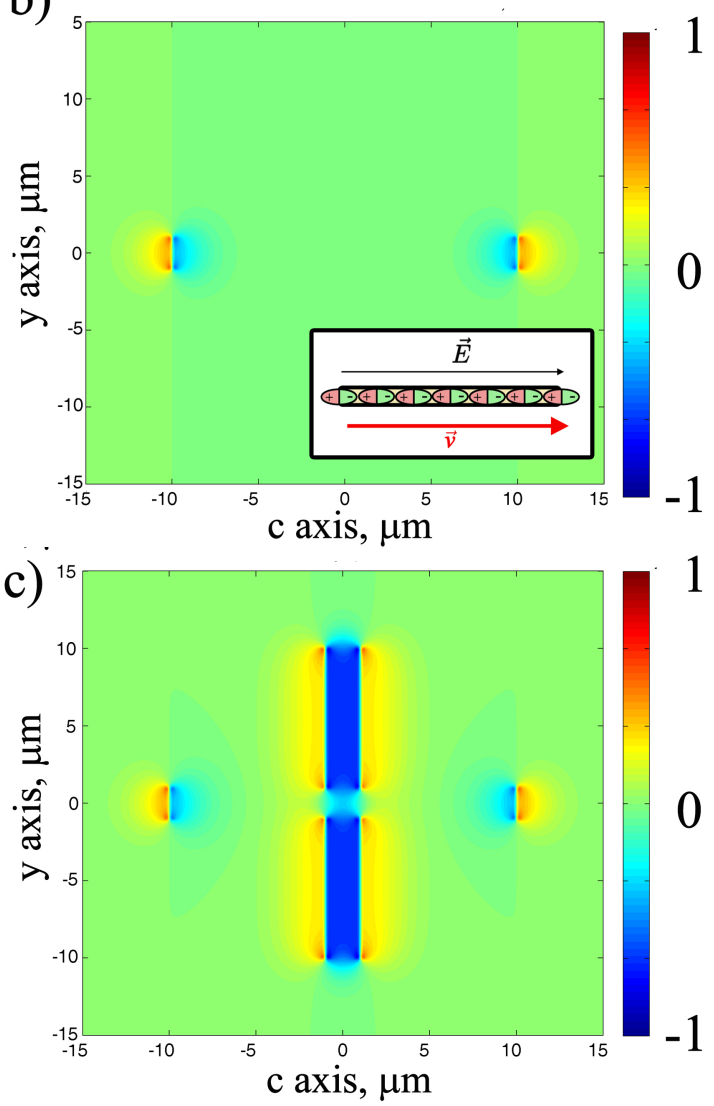

Fig. 2. Inducing photorefractive modifications via DLW. A change in refractive index when: a) straight line is recorded in perpendicular-to- $c$-axis direction; b) straight line is written in parallel-to- $c$-axis writing direction; c) two orthogonal lines are recorded. In last case, the refractive index change in middle section of perpendicular-to- $c$-axis written line is nullified with parallel-to$c$-axis line demonstrating data recording/erasure principle. Recording directions and internal space-charge field are depicted by $v$ and $\vec{E}$ in the scetches.

\section{Experimental setup}

Experimental setup used for the fabrication is depicted in Fig. 3. Femtosecond laser source ("Mai Tai" oscillator from "Spectra Physics") with a pulse duration $\sim 100 \mathrm{fs,} \mathrm{a} \mathrm{cen-}$ tral wavelength of $800 \mathrm{~nm}$ and a repetition rate of $80 \mathrm{MHz}$ was used for inscription tasks. The laser beam was attenu- ated with a variable attenuator and coupled into custom made DLW setup which consisted of high-reflecting dielectric mirrors for beam guiding, microscope objective (for beam focusing and sample imaging) and a stacked pair of highaccuracy translation stages for sample translation. The basis for DLW and imagining setup was Olympus IX-71 inverted microscope (with various numerical aperture (NA) objectives used for beam focusing) and two translation stage sets from PI ("Physik Instrumente"): for rough movement within (25 x 25) $\mathrm{mm}^{3}$ range model "M-686.D64" was used; and P$563.3 \mathrm{CD}$ for precision positioning in $(300 \times 300 \times 250) \mu^{3}$.

All recording process was controlled with the "3DPoli" (by "Femtika") software package.

We have used nominally pure congruent and Fe doped (500 ppm) near-stoichiometric ( $\mathrm{Li} / \mathrm{Nb}=49.85 / 50.15)$ LN samples. The samples were all side polished slabs with size of $(10 \times 10 \times 1) \mathrm{mm}^{3}$ in Y-cut orientation. Refractive indexes of $\mathrm{LN}$ are $n_{0}=2.3$ and $n_{e}=2.21$. From the absorption spectrum we see that both of these samples are transparent at $\lambda_{\text {laser }}=800 \mathrm{~nm}$ radiation, thus laser structuring should be attributed to two-photon or other non-linear absorption process occurring at the focus of laser beam.

The induced modifications were investigated by visible means with wide-field optical microscopy in phase contrast regime to asset the shape and size as well as to monitor regions of modified refractive index. Extended structures (diffractive constructions) were investigated by monitoring diffraction patterns and efficiencies of diffracted laser beam (we used specially shaped beam from $634 \mathrm{~nm}$ laser diode). In addition the interferometric measurements of modified refractive index region was carried out using static Michelson interferometer setup, where one arm was slightly tilted to produce interference fringe pattern on the CCD while the refractive index change were calculate from the shift of the fringes.

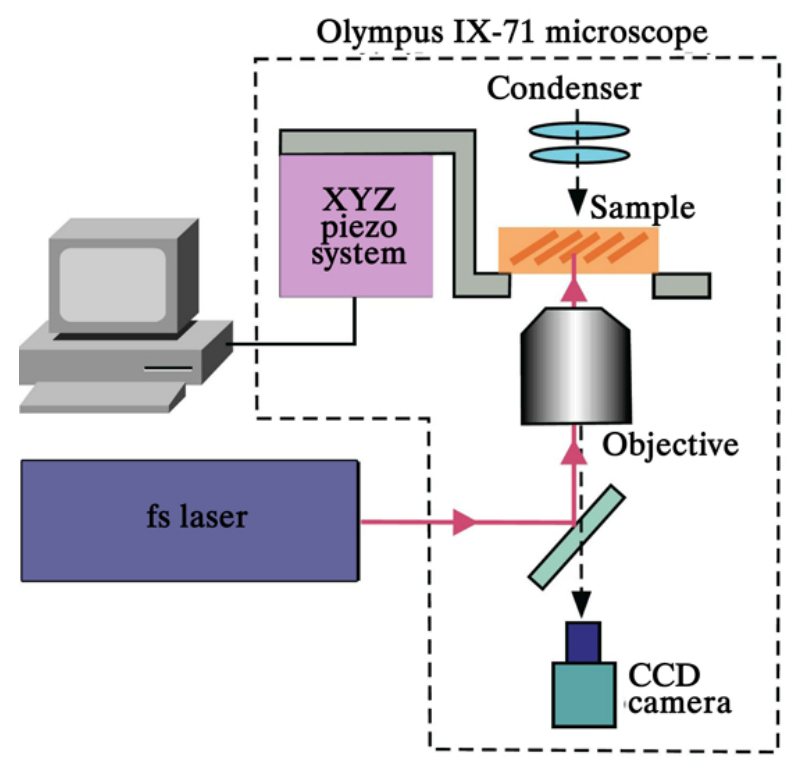

Fig. 3. Scetch of experimental DLW setup. 


\section{Results}

\subsection{Refractive index changes}

While focusing ultrashort laser beam in the volume of photorefractive crystal, the change in refractive index appears only in the vicinity of laser focus, where intensity requirements for nonlinear absorption are satisfied. Modification size can be tailored by laser intensity or by changing focusing conditions. The phase contrast images of modifications induced via single line inscription with different focusing objectives and laser powers in LN:Fe crystal are shown in Fig. 4.
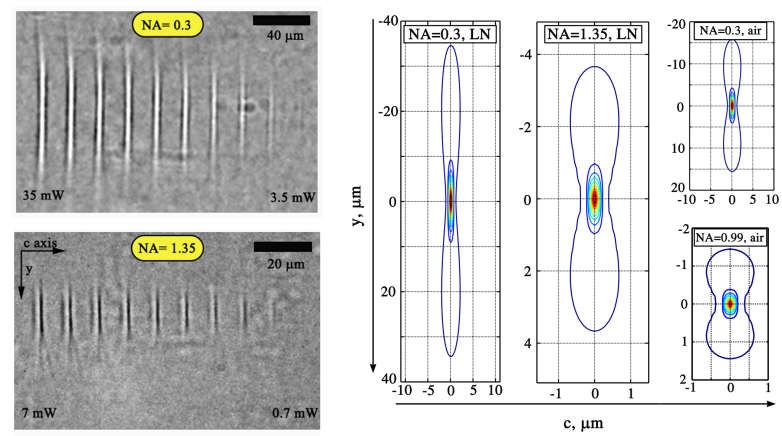

Fig. 4. Photoinduced modifications in Fe:LN: (left-top) Transverse view of modifications induced with $0.3 \mathrm{NA}$ objective with different laser powers (power decreases from left to right in 10 steps from $35 \mathrm{~mW}$ down to $3.5 \mathrm{~mW}$; line's length was $40 \mu \mathrm{m}$ in $x$ direction, translation velocity $20 \mu \mathrm{m} / \mathrm{s}$; recorded $\sim 290 \mu \mathrm{m}$ bellow the surface). (left-bottom) Transverse view of modifications induced with 1.35 NA oil immersion objective with different laser powers (power decreases from $7 \mathrm{~mW}$ down to $0.7 \mathrm{~mW}$; line's length $-40 \mu \mathrm{m}$ in $x$ direction, translation velocity $20 \mu \mathrm{m} / \mathrm{s}$; recorded $\sim 50 \mu \mathrm{m}$ bellow the surface)). (right) The intensity-squared distribution at the aberration-free focus in LN and air for different NA. Contours show regions with constant intensity-squared values. Due to mismatch of immersion-oil/LN refractive indexes, nominal value of $0.92 \mathrm{NA}$ was used instead of 1.35 NA for modeling.

A clear modification track is observed when the sample is translated perpendicularly to crystal's $c$-axis. The crosssection views of modifications (Fig. 4(left)) reveal highly elongated refractive index profile, where axial-to-lateral dimension ratios can reach up to 20:1 for lower NA values and up tot 7:1 for high NA immersion objective focusing. Typically in ultrashort glass micromodification sub-wavelength resolution can be reached in both directions [22], with quasispherical modification shape (so called "voxel") [23]. The reason for such elongation on our case is twofold: 1) high refractive index value of LN limits the use of traditional immersion oil objectives, as refractive index mismatch causes the appearance of spherical aberration; 2) photorefractive modification takes place at the intensities where critical free carrier density, capable to induce plasma absorption, is not reached; thus no plasma associated micro-defects such as voids or cracks develop. The region of photorefractive modification closely follows laser caustic that is modeled in Fig.4(right).
When high repetition rate lasers are used for modification recording it is more appropriate to use radiant exposure $(R)$ as the measure of energetic fabrication parameters. All modifications in our experiment were recorded in nonstationary conditions, which means that sample was always translated at $v$ velocity in respect to laser focus. The radiant exposure can be expressed as:

$$
R=k \frac{2 P}{\pi w_{0} v}
$$

here, $P$ is laser power, $w_{0}$ is the beam waist at the focus (at $1 / \mathrm{e}^{-2}$ level) and $k$ is the number of translation times though the same spot. Theoretical Gaussian beam waist value for $0.3 \mathrm{NA}$ and $800 \mathrm{~nm}$ is $0.8 \mu \mathrm{m}$ while for $0.45 \mathrm{NA}$ and $1.35 \mathrm{NA}$ (or non-immersion $0.92 \mathrm{NA}$ ) are $0.6 \mu \mathrm{m}$ and $0.4 \mu \mathrm{m}$, respectively. We have used these values to evaluate exposure, despite the fact that the resulted modifications were somewhat larger due to aberrations. This formula also implies that there is no difference on how total exposure is accumulated: either by increasing power, repetition number or translation velocity. This statement is not true in general; however in our case with used power ranges, we did not observed differences in how exposure was accumulated (i.e.using slow speed vs fast speed but multiple translations).

The magnitude of refractive index change was measured with Michelson interferometer: constant length lines $(200 \mu \mathrm{m})$ at different translation velocities were recorded in $\mathrm{LN}: \mathrm{Fe}$ and pure LN crystals and the change in refractive index was evaluated through the shift of the interference fringes. These results are shown in Fig. 5.

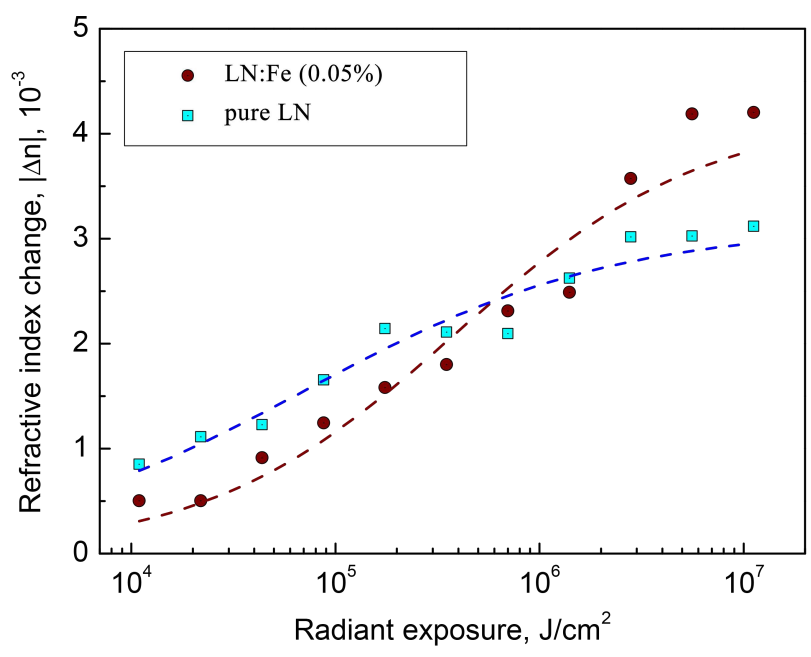

Fig. 5. Refractive index change on radiant exposure measured in pure LN and Fe:LN. Exposure was varied by increasing sample translation speed from $0.5 \mu \mathrm{m} / \mathrm{s}$ to $512 \mu \mathrm{m} / \mathrm{s}$ at constant laser power $(50 \mathrm{~mW})$; focused with $0.45 \mathrm{NA}$ objective, $150 \mu \mathrm{m}$ bellow the surface.

Only measurements for $e$-polarization are presented in the graph - measurement with $o$-polarized light give threetimes lower values, indicating that the refractive index change is of photorefractive origin. As expected, the buildup of refractive index change shows saturational behavior, and 
can be approximated with four-parameter logistic function (dashed curves showed in the graph), commonly used for exposure-dependent phenomena investigation. The maximum refractive index change at saturation was $(-4.2 \pm$ $0.1) \times 10^{-3}$ for $\mathrm{LN}: \mathrm{Fe}$, and $(-3.1 \pm 0.1) \times 10^{-3}$ for undoped LN. It is interesting that photorefractive index change under femtosecond laser pulses in doped and undoped crystals do not significantly differ - at lower exposure levels the refractive index change becomes even higher in pure crystal. This is a contrary result than expected for a low intensity radiation case. This shows that the presence of photoactive dopants are not essential for high refractive index change when charge separation is governed through two(multi)-photon absorption.

The BPVE saturation level is proportional to the donor/acceptor concentration, which explains the higher refractive index change values in doped crystal at greater exposure. Our achieved results are slightly larger, but comparable to these reported by others with intense laser radiation [24].

Local modification of refractive index is highly appealing for many photonics application as this is the key effect for integrated optics. Photorefractive index change has an advantage that it is not followed by any material deterioration which tends to produce scattering cracks limiting device performance.
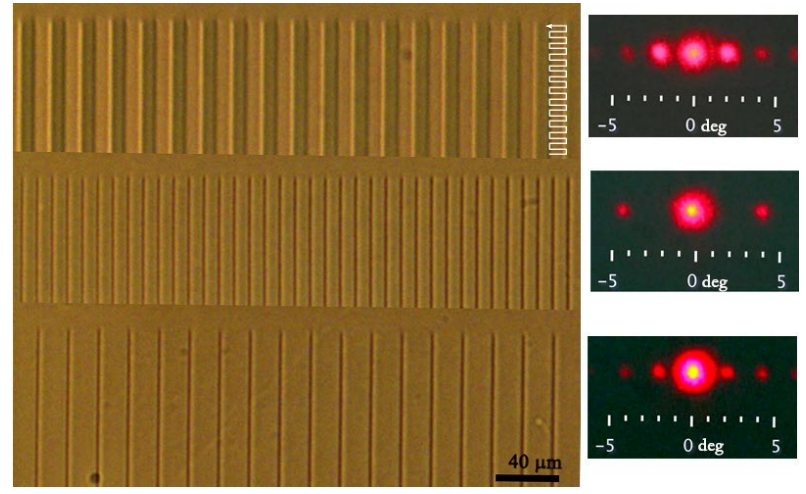

Fig. 6. a) Optical images of one-dimensional gratings with light diffraction patterns made in LN:Fe. Middle and bottom images depict gratings with periods of $10 \mu \mathrm{m}$ and $20 \mu \mathrm{m}$, respectively, while top grating is a square grating with period of $20 \mu \mathrm{m}$ (constructed from alternating zones of modified/non-modified areas each having $10 \mu \mathrm{m}$ diameter) made by raster patterning method (raster step $-1 \mu \mathrm{m}$ ). Recorded with $7.7 \times 10^{4} \mathrm{~J} / \mathrm{cm}^{2}$ exposure (laser power $20 \mathrm{~mW}$, translation velocity $-20 \mu \mathrm{m} / \mathrm{s}, 0.3 \mathrm{NA}$ objective).

A simple one dimensional grating inscribed in $\mathrm{LN}: \mathrm{Fe}$ is shown in Fig. 6-a) together with corresponding diffraction patterns. Grating grooves are formed by translating the sample perpendicularly to the crystal $c$-axis. In addition, the top image of Fig. 6-a) shows a $20 \mu \mathrm{m}$-period square grating where each groove has $10 \mu \mathrm{m}$ diameter made by raster patterning when the sample is translated along the $c$-axis (white zigzag trace in the image shows an inscription trajectory for a single groove). One can see that such patterning can produce discrete areas with uniformly modified refractive index without any signs of increased scattering. The diffraction pattern of the square grating clearly indicated that this grat- ing operates as a $20 \mu \mathrm{m}$ period grating (a line grating with such period is shown at the bottom of the image), but with increased diffraction efficiency. For comparison a grating with two times smaller period $(10 \mu \mathrm{m})$ with corresponding diffraction pattern is shown in the middle.

\subsection{Manipulation of recorded information}

The selective control of charge separation in photorefractive material via DLW makes it possible to implement dynamic data recording. This means that a single bit, represented by a line segment, can be recorded and selectively erased using the same DLW procedure, only by changing the recording direction. The experimental demonstration of this concept is shown in Fig.7.
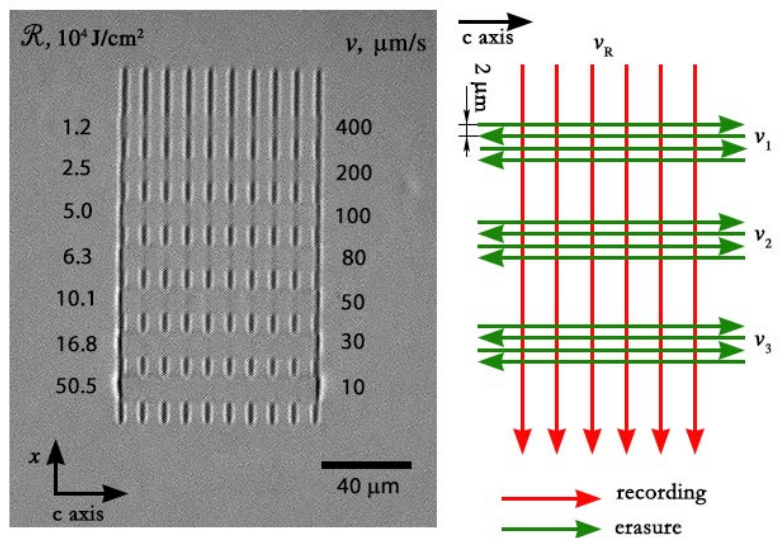

Fig. 7. A microscope image of recorded and erased information in LN:Fe. Sketch on the right depicts fabrication algorithm: lines are recorded with perpendicular to c-axis translating direction and later segments of the lines are erased by raster patterning consisting of lines separated by $2 \mu \mathrm{m}$ translated in parallel-to-c-axis direction. Line recording was carried out with the same parameters as in Fig. 6, while erasure parameters (exposure and corresponding translating velocity) are shown near erased segments (laser power used for erasure was $65 \mathrm{~mW}, 0.3 \mathrm{NA}$ ).

At first single lines are recorded in orthogonal-to- $c$-axis direction (red arrows). Later, segments of lines are erased by changing translation direction (green arrows). As was discussed in introductory part of this paper, topological charge separation is different when sample is translated along the $c$-axis with much lower intrinsic electric field and resulted refractive index modulation. One can see, that if sufficient erasure exposure is used, the previously recorded lines can be completely annihilated.

The same method can be realized for 3D data recording and erasing. To demonstrate this we recorded a thick grating with several stitched layers and, afterwards, a middle layer was erased by changing sample translation direction. The results are depicted in Fig 8-right.

Here we see the transverse image of thick grating in original condition and with erased segment. Note, that grating layers above and bellow erased area are intact, thus data in other layers is not affected by laser radiation. This clearly demonstrates the possibility to implement spatially selective data recording in 3D. Also, in Fig 8-right, the modified re- 
fractive index can be seen at the boundaries of erased region were erasure started/ended. At these points electric field is not homogeneous and changes in direction resulting in modulated refractive index which is clearly visible in phase contrast microscope images (as modeled in Fig. 2-b) .

Refractive index change is lower in the erased segment as electric field strength decreases in this region thus information re-recording becomes possible. However, in this case subsequent recording takes place in the presence of intrinsic electric field which reduces BPVE with drift currents and reduces the contrast of re-recorded information. We observed that recorded information can be read even after 50 recording/erasure cycles.

Global erasure of photorefractive information in entire crystal volume can be achieved with uniform visiblewavelength illumination or thermal annealing. All structures described in this paper were erased when sample was exposed for $1 \mathrm{~h}$ to defocused $10 \mathrm{~mW} / \mathrm{cm}^{2}$ intensity radiation from frequency doubled nanosecond Nd:YAG laser. The same result was achieved by keeping crystal at $100^{\circ} \mathrm{C}$ for $1 \mathrm{~h}$. After global erasure the same crystal can be used again for DLW inscription.

\section{Discussion}

DLW of photorefractive modifications opens some new opportunities not possible with traditional holographic recording technique. Binary patterns, square gratings, ordinary waveguides are challenging or even impossible to record via holography while they are simply realized with DLW as demonstrated in this paper. Techniques developed for holographic data storage in LN can be potentially applicable for DLW as both recording methods rely on the same physical phenomenon. One important issue is overall stability of recorded photorefractive modifications. We have found that structures recorded in $0.05 \% \mathrm{LN}: \mathrm{Fe}$ are still present after 6-months when storing at room temperature in the dark. Of course, when an integrated photonic device is used together with relatively intense radiation the local space-charge separation would be destroyed. This issue could be solved with thermal fixing which can make photorefractive modification permanent [25].

Several additional aspects of ultrafast DLW have also to be mentioned. In these experiments we report on modification induced with high repetition rate femtosecond oscillator. We have also tried to inscribe modifications with amplified laser systems (Ti:Sapphire at $1 \mathrm{kHz}$, and $\mathrm{Yb}: \mathrm{KGW}$ at $100 \mathrm{kHz}$ ), but the quality (visible contrast) of modifications was much worse. As the pulse repetition rate is much lower in these systems, one needs a higher pulse energy to reach equivalent exposure, which increases the possibility of crystal damage. Also nonlinear effects start to manifest which prevents tight beam focusing.

More complex DLW techniques could be implemented for application-orientate photorefractive modification tasks. For example modifications' long axial dimensions are beneficial for thick Bragg grating inscription, but not welcome in data recording as it reduces effective storage capacity. In the future it may be possible to suppress the axial elongation by recently proposed temporal focusing technique, which was successfully demonstrated in glass micromodifications [26].

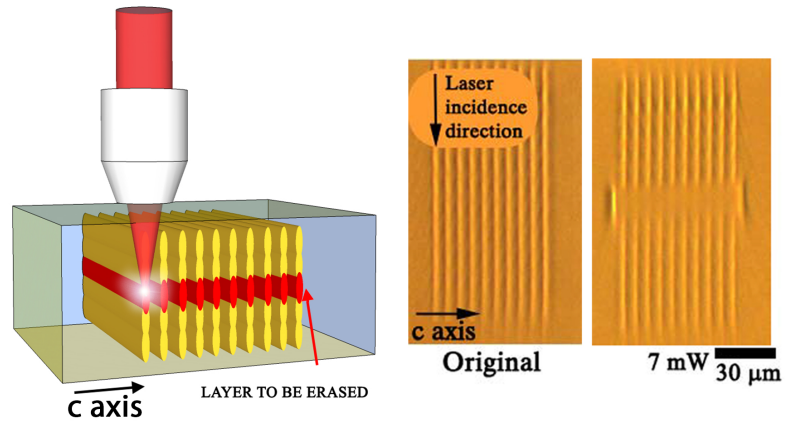

Fig. 8. (Right) A sketch of three-dimensional erasure: the several layers of grating are recorded on top of each other and later the middle layer is erased by changing translation direction. (Left) Three dimensional data erasure in LN:Fe crystal. Thick gratings were recorded perpendicular to $c$ axis (pulse average power $-7 \mathrm{~mW}$, speed $-20 \mu \mathrm{m} / \mathrm{s}$ ), and middle layer was erased by changing the writing direction to parallel to $c$-axis $(40 \mu \mathrm{m} / \mathrm{s}$ translation speed) Objective $-1.35 \mathrm{NA}$ with immersion oil.

\section{Conclusions}

We showed that DLW is versatile method for threedimensional photorefractive modification inscribing in irondoped and congruently pure lithium niobate crystal. Photorefractive effect can be exploited to create reversible structures with spatially tailored refractive index profile. Additionally, a dynamic data recording and erasure can be implemented with the same laser system. Also photorefractive index change magnitudes were evaluated, showing that saturation reaches $(-4.2 \pm 0.1) \times 10^{-3}$ for doped, and $(-3.1 \pm 0.1) \times 10^{-3}$ for pure crystals - a noticeably higher than usually achieved with longer laser pulses.

\section{References}

[1] L. Arizmendi: Phys. Status Solidi A, 201(2), (2004), 253.

[2] F. Agulló-López, G. Calvo, and M. Carrascosa: "Fundamentals of Photorefractive Phenomena", chapter in "Photorefractive Materials and Their Applications 1", (Springer, 2006).

[3] T. Volk and M. Wöhlecke: "Lithium Niobate: Defects, Photorefraction and Ferroelectric Switching", (Springer, 2009).

[4] F. Jermann and J. Otten: J. Opt. Soc. Am. B, 10(11), (1993), 2085.

[5] B. Sturman, M. Carrascosa, and F. Agullo-Lopez: Phys. Rev. B, 78, (2008), 245114.

[6] F. Lüdtke, N. Waasem, K. Buse, and B. Sturman: Appl. Phys. B, 105(1), (2011), 35.

[7] K. M. Davis, K. Miura, N. Sugimoto, and K. Hirao: Opt. Lett., 21(21), (1996), 1729.

[8] R. Gattass and E. Mazur: Nat. Photonics, 2(4), (2008), 219.

[9] L. Gui, B. Xu, and T. Chong: IEEE Photonics Technol. Lett., 1(5), (2004), 1337. 
[10] R. Thomson, S. Campbell, I. Blewett, A. Kar, and D. Reid: Appl. Phys. Lett., 88, (2006), 111109.

[11] J. Burghoff, H. Hartung, S. Nolte, and A. Tuennermann: Appl. Phys. A, 86(2), (2007), 165.

[12] F. Chen and J. R. V. de Aldana: Laser Photonics Rev., 8(2), (2014), 251.

[13] D. Paipulas, V. Kudriašov, M. Malinauskas, V. Smilgevičius, and V. Sirutkaitis: Appl. Phys. A, 104(3), (2011), 769.

[14] P. Sivarajah, C. Werley, B. Ofori-Okai, and K. Nelson: Appl. Phys. A, 112(3), (2013), 615.

[15] D. Deshpande, A. Malshe, E. Stach, V. Radmilovic, D. Alexander, D. Doerr, and D. Hirt: J. Appl. Phys., 97(7), (2005), 074316.

[16] O. Beyer, I. Breunig, F. Kalkum, and K. Buse: Appl. Phys. Lett., 88(5), (2006), 051120.

[17] E. G. Gamaly, S. Juodkazis, V. Mizeikis, H. Misawa, A. V. Rode, and W. Krolikowski: Phys. Rev. B, 81(5), (2010), 054113.
[18] S. Juodkazis, M. Sudzius, V. Mizeikis, H. Misawa, E. G. Gamaly, Y. Liu, O. A. Louchev, and K. Kitamura: Appl. Phys. Lett., 89(6), (2006), 062903.

[19] V. Fridkin: Crystalogr. Rep+, 46(4), (2001), 654.

[20] K. Buse and K. H. Ringhofer: Appl. Phys. A, 57, (1993), 161.

[21] N. U. K. Onuki and T. Saku: J. Opt. Soc. Am., 62(9), (1972), 1030.

[22] E. N. Glezer and E. Mazur: Appl. Phys. Lett., 71(7), (1997), 882.

[23] G. Zhou and M. Gu: Appl. Phys. Lett., 87(24), 241107.

[24] O. Althoff, A. Erdmann, L. Wiskott, and P. Hertel: Phys. Stat. Sol.(a), 128(1), (1991), K41.

[25] K. Buse, S. Breer, K. Peithmann, S. Kapphan, M. Gao, and E. Krätzig: Phys. Rev. B, 56, (1997), 1225.

[26] F. He, H. Xu, Y. Cheng, J. Ni, H. Xiong, Z. Xu, K. Sugioka, and K. Midorikawa: Opt. Lett., 35(7), (2010), 1106.

(Received: June 17, 2015, Accepted: June 13, 2016) 